\title{
Spectrum of liver dysfunction in patients with dengue infection and the markers of severe disease: study from a tertiary care centre in Punjab
}

\begin{abstract}
Introduction: Dengue is a major international health concern that is prevalent in tropical and sub tropical countries. Study of dengue infection and its Liver complications are scarce from countries like India. This study was done to assess the frequency and spectrum of liver dysfunction in Dengue infection patients.
\end{abstract}

Patients and Methods: A hospital based record analysis study was performed at a tertiary care centre. All the inpatients (>18years) who were diagnosed with dengue infection between January 2015 to May 2017 were included in this study. A total of 281 patients were included in the study.

Results: Patients were classified as classical dengue fever (DF) $88.3 \%$ dengue haemorrhagic fever (DHF) 7.5\% and dengue shock syndrome (DSS) $4.3 \%$. The mean age was $43.13(+15.50)$ years and male: female ratio was $2: 1$. Deranged serum glutamicoxaloacetic transaminase (SGOT) and/or Serum glutamic pyruvic transaminase (SGPT) was present in $98.9 \%$ of patients .The mean Total Bilirubin, SGOT, SGPT, albumin, ALP (alkaline phosphatase) and INR (International Normalized Ratio) values were $0.95 \mathrm{mg} / \mathrm{dl}, 687.28 \mathrm{U} / \mathrm{L}, 293.65 \mathrm{U} / \mathrm{L}, 3.71 \mathrm{~g} / \mathrm{dl}, 112.14 \mathrm{U} / \mathrm{L}$ and $1.30 \mathrm{respectively}$. The mean value of SGOT was significantly higher than SGPT. The degree of rise of SGOT, SGPT, INR, Bilirubin and ALP was significantly more in DHF and DSS, as compared to DF. 28 patients $(10 \%)$ had evidence of coagulopathy (INR $>1.5)$ and 6 patients had evidence of hepatic encephalopathy.

Conclusion: Liver dysfunction in the form of raised SGOT/SGPT was seen in almost all patients with rise in SGOT significantly more than SGPT. Preferentially high SGOT may serve as an early indicator of dengue infection while high values of bilirubin, SGOT, SGPT, ALP and INR may be an indicator of severe disease and poor prognosis.
Volume 3 Issue 4 - 2017

\author{
Amit Soni,' Parth M Patel, ${ }^{2}$ Nirmaljeet Singh \\ Malhi,' Gurcharan Lal Avasthi \\ 'Department of Gastroenterology and liver diseases, SPS \\ Hospital, India \\ ${ }^{2}$ Department of Medicine, DNB Resident Medicine, SPS \\ Hospital, India
}

Correspondence: Nirmaljeet Singh Malhi, Senior Consultant and Head, Department of Gastroenterology and liver diseases, SPS Hospital, Sherpur chownk, Ludhiana, Pin- 141003, India, Tel 0887205 4262, Email drnjsmalhi@gmail.com

Received: August 06, 2017 | Published: September II, 2017

Keywords: dengue infection, liver, bilirubin, heart failure, acute viral hepatitis

Abbreviations: DSS, dengue shock syndrome; DF, dengue fever; SGOT, serum glutamic-oxaloacetic transaminase; SGPT, serum glutamic pyruvic transaminase; INR, international normalized ratio; ALP, alkaline phosphatase; DHF, dengue hemorrhagic fever; NAFLD, non-alcoholic fatty liver disease

\section{Introduction}

At present, Dengue fever is the most common cause of arboviral disease worldwide. It is one of the most important causes of febrile illness in India and other tropical countries. Almost 2.5billion people in 100 endemic countries are believed to be susceptible, so are the large numbers of travelers to these endemic countries. Various factors have been implicated for increasing disease burden in tropics and spread of disease globally. These factors include poor hygiene, broken health system and booming international travels. ${ }^{1,2}$ Dengue virus was isolated in India for the first time in 1945. The first evidence of occurrence of dengue fever in the country was reported in 1956 from Vellore district in Tamil Nadu. The first dengue hemorrhagic fever (DHF) outbreak occurred in Calcutta (West Bengal) in 1963. ${ }^{3}$ Dengue is prevalent throughout India in urban as well as rural areas. According to latest data there are 64058 cases and 135 deaths from dengue in 2015 (till 25th October 2015). As per a study, the total estimated direct annual medical cost for Dengue in India was US \$548million. Ambulatory settings treated $67 \%$ of cases representing $18 \%$ of costs, whereas $33 \%$ of cases were hospitalized, comprising $82 \%$ of costs. ${ }^{4}$

Majority of the patients infected with dengue virus are asymptomatic $(80 \%)$ or have mild symptoms such as fever. Few have more severe illness $(5 \%)$ and in a small proportion it is lethal. ${ }^{5,6}$ Liver involvement in the form of abnormalities in liver function test is commonly seen in dengue fever. The liver function abnormalities vary from mild elevation in transaminase and bilirubin to a picture mimicking viral hepatitis or acute liver failure $(\geq 10 \mathrm{X}$ transaminase, deranged INR). In most of the cases the liver involvement remains asymptomatic but jaundice and acute liver failure has also been very well seen in patients with severe dengue. ${ }^{7}$ Hepatic dysfunction is caused by a direct effect on liver cells or as a consequence of deranged host immune response against the virus. ${ }^{8}$ Other factors including preexisting liver damage and the use of hepatotoxic drugs etc may also play a role. ${ }^{9}$ Elevated liver enzymes in dengue are an early marker of dengue infection. It is also a predictor for assessing the disease severity. ${ }^{10}$ The number of patients affected by the virus is increasing each year however few studies have been done in this area. Little data is available on liver involvement in dengue fever among adults and very scanty studies have been done in northern India/Punjab on this subject. 


\section{Patients and methods}

A hospital based record analysis study was performed at a tertiary care centre. All the in patients (>18years) who were diagnosed with dengue infection between January 2015 to May 2017 were included in this study. History, clinical features, Investigation and treatment given was collected from the records. Patients with pre existing or concurrent liver disease due to any other etiology were excluded from the study (e.g. diagnosed Chronic Liver Disease co- existing or recent history of infectious disease like acute viral hepatitis, malaria, typhoid, leptospirosis, history of intake of any hepatotoxic drugs, history of alcoholic liver disease, history of Non-alcoholic Fatty Liver Disease (NAFLD) Wilson's disease, autoimmune hepatitis and heart failure etc.). A total of 281 patients were included in the study. Patients were categorized in to classical dengue fever (DF), Dengue hemorrhagic fever, Dengue shock syndrome as per WHO criteria. ${ }^{11}$ Dengue infection was detected using Rapid test (Immunochromatography) for Dengue NS1 Antigen/IgM Dengue. Few were confirmed using ELISA for IgM Dengue.

\section{Statistical analysis}

Results for continuous variables were expressed as means and standard deviation. Categorical variables were expressed as percentages. Student's t-test for continuous variables and Chi square test for discrete variables were used to test significance. The $p$ value of less than 0.05 was considered statistically significant. The SPSS 22 software was used for statistical analysis.

\section{Results}

Mean age of patients in our study was 43.13years $( \pm 15.50)$ and male is to female ratio was $2: 1.248$ patients $(88.3 \%)$ were diagnosed to have dengue fever (DF) while 21 patients $(7.5 \%)$ were classified as Dengue hemorrhagic fever (DHF) and 12 patients (4.3\%) as Dengue shock syndrome (DSS). A vast majority of patients (98.9\%) had at least one of the two enzymes level (SGOT/SGPT) elevated above the upper limit of normal $(>40 \mathrm{IU} / \mathrm{L})$. Mean values of various liver function test is shown in Table 1.

Table I Mean values of various liver function tests

\begin{tabular}{lll}
\hline Lab parameters(units) & Mean values & SD(standard deviation) \\
\hline T. Bilirubin (mg/dl) & 0.95 & 0.72 \\
SGOT(U/L) & 687.28 & 3037.94 \\
SGPT(U/L) & 293.65 & 1041.89 \\
S.Albumin (g/dl) & 3.39 & 0.45 \\
S.Alkaline Phosphatase (U/L) & $1 \mathrm{I} 2.14$ & 82.17 \\
INR & 1.3 & 0.28 \\
\hline
\end{tabular}

Mean value of SGOT was significantly higher than SGPT (Figure 1). Majority of patients had their SGOT values between 3 to 10times upper limit of normal (45.6\%) while for SGPT majority values were between 1 to 3times upper limit of normal (Table 2). In contrast to SGOT/SGPT, a scanty number of patients had elevation in Bilirubin. 10 patients $(3.6 \%)$ had elevation in Bilirubin more than $3 \mathrm{mg} / \mathrm{dl}$. 28 patients $(10 \%)$ had evidence of coagulopathy (INR $>1.5)$ and 6 patients were found to have hepatic encephalopathy. Almost $4 / 5$ th of patients $(79.4 \%)$ had platelet counts $<1$ lakh at some point of time during hospital stay. A meagre percentage (2.1\%) had leukocytosis, while rest had either normal TLC or leucopenia. Comparison of laboratory parameters was done between male and female patients (Table 3). Also Comparison of various liver function tests was done between DF, DHF (Table 4).

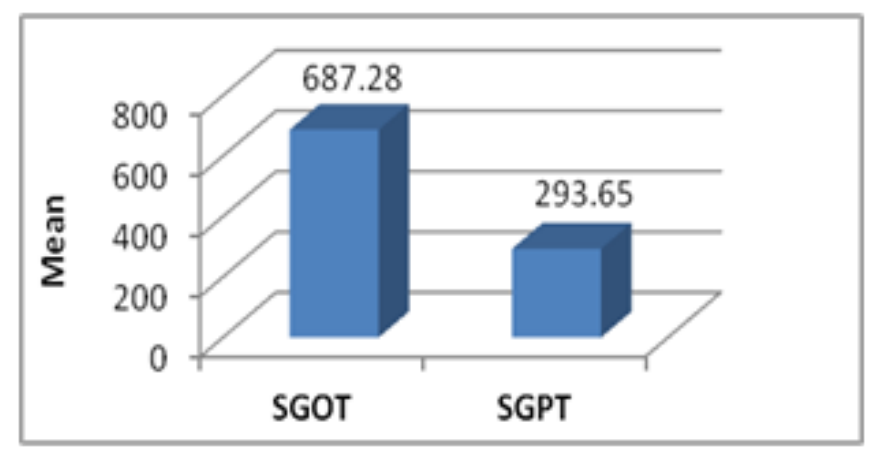

Figure I Comparison of mean value of SGOT \& SGPT.

Table 2 Percentage of patients who have various level of SGOT \& SGPT elevation

\begin{tabular}{lll}
\hline SGOT/SGPT(U/L) & Frequency & Percent \\
\hline$<40$ & 3 & 1.1 \\
$>40$ & 278 & 98.9 \\
Total & $28 \mid$ & 100 \\
SGOT(U/L) & Frequency & Percent \\
$<40$ & 4 & 1.4 \\
$4 \mid-120$ & 128 & 45.6 \\
I2I-400 & 103 & 36.7 \\
$>400$ & 46 & 16.4 \\
Total & $28 \mid$ & 100 \\
SGPT(U/L) & Frequency & Percent \\
$<40$ & $1 \mid$ & 3.9 \\
$4|-| 20$ & 78 & 27.8 \\
I2I-400 & 167 & 59.4 \\
$>400$ & 25 & 8.9 \\
Total & $28 \mid$ & 100 \\
\hline
\end{tabular}

Table 3 Comparison of various parameters between male and female Dengue patients

\begin{tabular}{|c|c|c|c|c|c|}
\hline \multirow{2}{*}{ Characteristic } & \multicolumn{2}{|l|}{ Male } & \multicolumn{2}{|l|}{ Female } & \multirow{2}{*}{ p-value } \\
\hline & Mean & SD & Mean & SD & \\
\hline Age( in Years) & 42.799 & $|5.8| \mid$ & 43.804 & 14.93 & 0.611 \\
\hline $\begin{array}{l}\text { Platelets(per } \\
\text { cu.mm) }\end{array}$ & 0.554 & 0.363 & 0.981 & 3.711 & 0.118 \\
\hline T. Bilirubin(mg/dl) & 0.959 & 0.663 & 0.933 & 0.835 & 0.774 \\
\hline SGOT(U/L) & 720.603 & 3517.682 & 618.837 & 1681.11 & 0.793 \\
\hline SGPT(U/L) & 318.443 & 1230.32 & 242.717 & 457.187 & 0.568 \\
\hline S.Albumin(g/dl) & 3.729 & 3.04 & 3.668 & 3.433 & 0.881 \\
\hline $\begin{array}{l}\text { S.Alkaline } \\
\text { Phosphatase(U/L) }\end{array}$ & 107.106 & 76.895 & 122.478 & 91.646 & 0.141 \\
\hline INR & 1.294 & 0.253 & 1.327 & 0.335 & -0.913 \\
\hline
\end{tabular}


Table 4 Comparison of biochemical liver test derangements between various Dengue group

\begin{tabular}{|c|c|c|c|c|c|c|}
\hline \multirow{2}{*}{ Characteristic } & \multicolumn{3}{|l|}{ Disease } & \multirow{2}{*}{$\begin{array}{l}\text { DF vs DHF } \\
\text { p value }\end{array}$} & \multirow{2}{*}{$\begin{array}{l}\text { DF vs DSS } \\
\text { p value }\end{array}$} & \multirow{2}{*}{$\begin{array}{l}\text { DHF vs DSS } \\
\text { p value }\end{array}$} \\
\hline & DF & DHS & DSS & & & \\
\hline No of Patients & 248 & 21 & 12 & & & \\
\hline Mean SGOT(U/L) & 218.528 & 1747.762 & 8519.083 & 0.008 & 0 & 0 \\
\hline Mean SGPT(U/L) & 125.805 & 856.429 & 2777.583 & 0 & 0 & 0 \\
\hline Mean S.Albumin(gm\%) & 3.735 & 4.01 & 2.65 & 0.704 & 0.248 & 0.237 \\
\hline Mean Bilirubin(mg/dl) & 0.78 & 2.01 & 2.69 & 0 & 0 & 0 \\
\hline Mean INR & 1.25 & 1.449 & 2.19 & 0 & 0 & 0 \\
\hline
\end{tabular}

\section{Discussion}

In this hospital based study the spectrum and frequency of liver function tests was studied in 281 Dengue infected patients. In this study most of the patients $(88.3 \%)$ had classical Dengue fever while DHF and DSS were present in $7.5 \%$ and $4.3 \%$ patients respectively. The results are similar to a study from Delhi where in Dengue fever was seen in majority while DHF and DSS were seen in $9.3 \%$ and $2.2 \%$ of the total cases respectively. ${ }^{12}$ Liver dysfunction in the form of Transaminasemia (SGOT/SGPT>ULN) was found ubiquitously (98.9\%) in our study. Shukla et al. ${ }^{13}$ found that $100 \%$ patients had an elevated SGOT and 91\% had elevated SGPT among patients with dengue. Similar form of raised SGOT/SGPT has been documented in other studies. ${ }^{14,15}$ In contrast to SGOT/SGPT, raised level of bilirubin or ALP was seen in few patients. We found that mean value of SGOT was more than double the value of SGPT. This differs from the pattern seen in viral hepatitis where SGPT values are higher than SGOT. The average value of SGOT were found to be higher than SGPT in study from Kolkata (India) and Karachi (Pakistan). ${ }^{16,17}$ The exact cause of this is uncertain, but it has been suggested that it may be due to excess release of AST from damaged monocytes during dengue infection. ${ }^{8}$ This pattern of SGOT/SGPT may hint towards dengue infection when the presentation and laboratory parameters mimic Acute Viral Hepatitis.

Comparison of the lab parameters was done between male and female patients but there was no significant difference in liver function tests. Similar to our results, a large study from Brazil also found that there was no significant difference in the level of transaminase elevation between male and female but contrary to our observation they found that the liver damage was more common among females. ${ }^{18}$ Moreover very few studies have compared the parameters between male and female patients. We observed statistically significant difference when liver function tests were compared between DF, DHF and DSS. Values of Bilirubin, SGOT, SGPT ALP and INR among DHF was significantly higher ( $p$ values $<0.05$ ) when compared with DF but albumin levels were comparable. (p value $=0.704$ ). Similarly patients with DSS had liver function tests (Bilirubin, SGOT, SGPT, ALP, INR) which was higher Vs DF ( $p$ values $<0.05$ ). In this group also albumin value was comparable ( $p$ value $=0.248$ ). This clearly indicates that with increasing disease severity the severity of hepatic dysfunction also increase. The finding is of great clinical significance as this indicates that higher values of SGOT, SGPT, Bilirubin, ALP and INR are seen in patients with severe disease. So in a way grossly deranged LFT's can point towards severe form of dengue. Liver injury has been proposed to be a good positive predictive factor for the development of DHF. ${ }^{19}$ Kuo et al. ${ }^{20}$ reported in their study that higher levels of aminotransferases were related to increased severity of the disease. Study by Chhina et al. ${ }^{21}$ had a very similar finding to our study. They noted a greater degree of hepatic injury in the DHF group (significantly higher values of SGOT and ALP) and DSS group (significantly higher values of all biochemical liver tests) as compared to the DF group, suggesting that the degree of liver injury may be related to the severity of dengue infection. Albumin is usually affected in chronic liver disease but dengue is an acute illness so albumin level remains stable. The appearance of jaundice in cases of DF/DHF/DSS may be multifactorial. It can be due to hepatic injury caused by the dengue virus and or hypoxia and tissue ischemia in cases of dengue shock. ${ }^{8}$ Jaundice occur more in complicated than in uncomplicated cases. Jaundice in dengue infection have been associated with fulminant hepatic failure and it itself is a poor prognostic sign. ${ }^{22}$ In our study $3.6 \%(10 / 281)$ patients were found to have total bilirubin $>3 \mathrm{mg} / \mathrm{dl}$.

Hepatic encephalopathy was seen in only six patients in our study. LFT abnormalities in these patients were significantly higher from those without encephalopathy, indicating that liver failure was the cause of altered sensorium in these patients. Other possible reasons for the neurological symptoms in dengue infection are metabolic acidosis, severe disseminated intravascular coagulation, gross hemorrhage or edema in the brain, or hyponatremia due to excessive fluid administration. Abnormal PT indicates abnormal coagulopathy. It is seen more frequently in cases of dengue presenting with acute hepatic failure, DIC, shock and hepatic encephalopathy. In cases with severe dengue because of increased hepatic cell damage would have caused decreased production of clotting factors resulting in prolonged PT. ${ }^{23}$ In our study, prolonged prothrombin time was observed significantly higher in dengue shock cases in comparison to classical dengue fever and dengue hemorrhagic fever. There is little limitation in this study. As it is a hospital based record analysis study so it is not true representation of all dengue infection .Majority of dengue infections are treated on outpatient basis and only few who are very symptomatic or have grossly deranged laboratory parameters are treated in hospital. Patients who were positive with rapid test (Immunochromatographic test) were also included in this study though ELISA IgM dengue is the best and recommended test for dengue infection confirmation.

\section{Conclusion}

We report the liver function test abnormalities in a large group of patients with dengue infection. Liver dysfunction in the form of raised SGOT/SGPT was seen in almost all patients with rise in SGOT significantly more than SGPT. Values of SGOT/SGPT, ALP, INR and bilirubin were significantly higher in DHF and DSS Vs DF. Therefore, 
while preferentially high SGOT may serve as an early indicator of dengue infection, while high values of bilirubin, SGOT, SGPT and ALP may be as indicator of severe disease and poor prognosis.

\section{Acknowledgements}

None.

\section{Conflict of interest}

Author declares that there is no conflict of interest.

\section{References}

1. Guzman MG, Halstead SB, Artsob H, et al. Dengue: a continuing global threat. Nat Rev Microbiol. 2010;8(12 Suppl):S7-S16.

2. Guzmán MG, Kourí G. Dengue: an update. Lancet Infect Dis. 2002;2(1):33-42.

3. Baruah K, Dhariwal AC. Epidemiology of dengue, its prevention and control in India. J Indian Med Assoc. 2011;109(2):82-86.

4. Shepard DS, Halasa YA, Tyagi BK, et al. Economic and Disease Burden of Dengue Illness in India. Am J Trop Med Hyg. 2014;91(6):1235-1242.

5. Whitehorn J, Farrar J. "Dengue". Br Med Bull. 2010;95:161-173.

6. Reiter P. Yellow fever and Dengue: a threat to Europe? Euro Surveill. 2011;15(10):19509.

7. Samanta J, Sharma V. Dengue and its effects on liver. World J Clin Cases. 2015;3(2):125-131.

8. Fernando S, Wijewickrama A, Gomes L, et al. Patterns and causes of liver involvement in acute dengue infection. BMC Infect Dis. 2016;16:319.

9. Ageep AK. Degree of liver injury in Dengue virus infection. Journal of General and Molecular Virology. 2012;4(1):1-5.

10. Seneviratne SL, Malavige GN, De Silva HJ. Pathogenesis of liver involvement during dengue viral infections. Trans $R$ Soc Trop Med Hyg. 2006;100(7):608-614.
11. World Health Organization (WHO). Dengue haemorrhagic fever: diagnosis, treatment, prevention and control. 2nd ed. Geneva: World Health Organization; 1997.

12. Makroo RN, Raina V, Kumar P, et al. Role of platelet transfusion in the management of dengue patients in a tertiary care hospital. Asian $J$ Transfus Sci. 2007;1(1):4-7.

13. Shukla V, Chandra A. A Study of Hepatic Dysfunction in Dengue. $J$ Assoc Physicians India. 2013;61(7):460-461.

14. Daniel R, Rajamohanan, Philip AZ. A study of clinical profile of dengue fever in Kollam, Kerala, India. Dengue Bulletin. 2015;29:197-202.

15. Itha S, Kashyap R, Krishnani N, et al. Profile of liver involvement in dengue virus infection. Natl Med J India. 2005;18(3):127-130.

16. Parkash O, Almas A, Jafri SM, et al. Severity of acute hepatitis and its outcome in patients with dengue fever in a tertiary care hospital Karachi, Pakistan (SouthAsia). BMC Gastroenterol. 2010;10:43.

17. Bandyopadhyay D, Chattaraj S, Hajra A, et al. A Study on Spectrum of Hepatobiliary Dysfunctions and Pattern of Liver Involvement in Dengue Infection. J Clin Diagn Res. 2016;10(5):OC21-OC26.

18. Souza LJ, Alves JG, Nogueira RM, et al. Aminotransferase changes and acute hepatitis in patients with dengue fever: analysis of 1,585 cases. Braz J Infect Dis. 2004;8(2):156-163.

19. De Souza LJ, Gonçalves Carneiro H, Souto Filho JT, et al. Hepatitis in dengue shock syndrome. Braz J Infect Dis. 2002;6(6):322-327.

20. Kuo CH, Tai DI, Chang-Chien CS, et al. Liver biochemical tests and dengue fever. Am J Trop Med Hyg. 1992;47(3):265-270.

21. Chhina, Rajoo Singh Goyal, Omesh Chhina, et al. Liver function tests in patients with dengue viral infection. Dengue Bulletin. 2008;32:110-117.

22. Mourão MP, Lacerda MV, Bastos Mde S, et al. Dengue haemorrhagic fever and acute hepatitis: a case report. Braz J Infect Dis. 2004;8(6):461-464.

23. Jagadish K, Patwari AK, Sarin SK, et al. Hepatic manifestations in typhoid fever. Indian Pediatr. 1994;31(7):807-811. 